\title{
LA HOMOSEXUALIDAD EN EL TIEMPO
}

\author{
HOMOSEXUALITY IN TIME
}

\section{Douglas Verbicaro Soares}

Universidade Federal de Roraima, Boa Vista, RR, Brasil. E-mail: douglas_verbicaro@yahoo.com.br

\author{
DOI: https://doi.org/10.46550/amormundi.v2i2.46
}

Recebido em: 18.12.2020

Aceito em: 17.02.2021

\begin{abstract}
Resumen: El presente estudio busca la sensibilización social en diferentes sociedades con el objetivo de promocionar discusiones sobre cuestiones de diversidad sexual y no discriminación. Así, servirá como alternativa para combatir la exclusión social de personas homosexuales, siendo fundamental para integrar ese colectivo, victimado en la historia de la humanidad por siglos. La estructura del estudio ha sido pensada para visibilizar realidades de prejuicio y estigmatización de los homosexuales en el tiempo. Para la investigación ha sido empleada una metodología basada en la fuente bibliográfica multidisciplinar. El artículo concluye por la existencia de prácticas discriminatorias hacia a las personas homosexuales hasta la actualidad.
\end{abstract}

Palabras claves: Sexualidad. Historia. Discriminación.

Abstract: This study seeks social awareness in different societies with the aim of promoting discussions on issues of sexual diversity and non-discrimination. Thus, it will serve as an alternative to combat the social exclusion of homosexual people, being fundamental to integrate this collective, victimized in the history of humanity for centuries. The structure of the study has been designed to make realities of prejudice and stigmatization of homosexuals visible over time. A methodology based on the multidisciplinary bibliographic source has been used for research. The article concludes with the existence of discriminatory practices towards homosexual people.

Keywords: Sexuality. History. Discrimination.

\section{Introducción}

Zl desarrollo de nuestro razonamiento comenzará con un enfoque histórico sobre Cla orientación sexual homosexual, haciendo hincapié en que las influencias de los pensamientos basados en estereotipos, prejuicios y comportamientos discriminatorios, que contribuyeron para la estigmatización de las personas homosexuales de una manera verdaderamente negativa (extendida en el tiempo).

Estos eventos convirtieron a los personajes como personas de la segunda categoría (los homosexuales) y que fueron expulsados de la participación social, política, religiosa, económica y de muchas otras formas de exclusión en distintas sociedades. Sin olvidar de destacar que estas personas fueron olvidadas durante mucho tiempo, como por ejemplo el Estado brasileño, a través 
de políticas públicas no eficaces que les no permitirían superar las situaciones de vulneración que enfrentan el colectivo de homosexuales.

Así, la presente investigación tiene por objetivo discutir sobre la orientación sexual homosexual, destacando que nada más es que una de las diversidades sexuales existentes. Así, para que su protección estuviera compaginada con las ideas de los Derechos Humanos, como pueden ser: derecho a la vida, a la dignidad humana y a la posibilidad de que cada persona pueda desarrollarse plenamente como persona y ejercer sus humanidades, sin las adversidades que pueden obstaculizar la superación y, también, permitir que estas personas se integren verdaderamente en la sociedad en la que viven.

Bajo este punto de mira, creemos que el uso y la asistencia de diversas ciencias servirían para dar un enfoque multidisciplinar al tema trabajado de la homosexualidad. Por esta razón, la ayuda de las ciencias jurídicas, la pedagogía, la psicología, la medicina, la historia contribuyó para el desarrollo de la presente investigación. El estudio ha sido dividido en 2 (dos) partes, más la introducción, consideraciones finales y referencias.

Algunas preguntas han sido pensadas para la investigación: ¿Lo que dice la historia sobre la homosexualidad? ¿Cómo ha sido la persecución a los homosexuales en el tiempo?

\section{La previsión sobre la homosexualidad en la historia}

El período final de la Edad Media ha sido marcado por los deseos de restablecer el contacto con las ideas del período de la antigüedad clásica, motivados por la expresión de distintos grupos de pensadores, los llamados humanistas. Ese movimiento ha sido desarrollado en Florencia, en el Siglo XIV, con un papel destacado a Coluccio Salutati: que era secretario de Cartas latinas de la Señoría y amigo personal de Petrarca y de Boccaccio (GARCÍA VALDÉS, 1981, p. 52). Estos mismos poetas colaboraron con él en el interés por el griego y por el latín y por el afán de coleccionar las obras clásicas. Ese período histórico simbolizó un fuerte regreso a Cultura del modelo clásico, es decir, lo que había sido desarrollado tanto por los griegos, como también por sus sucesores, los romanos. Que durante el tiempo medieval, habían sido dejados a según plano y guardados, conservados en los monasterios, por sus monjes, pasando por una traducción por los árabes. Siendo posteriormente buscados por lo que representó el helenismo, con sus influencias a las civilizaciones paganas y su modelo imitado (GARCÍA VALDÉS, 1981, p. 53-4).

Para el autor Zanotti, más específicamente en el siglo XV: habló sobre el ideal en la Grecia antigua, cuanto a la interpretación dada al amor entre dos hombres, es decir, el amor por la belleza corporal y material de un hombre joven, se llegaría a la idea de la belleza ideal. Para ese momento histórico, Zanotti nos recuerda el mito de Ganimedes, un joven pastor, que era hijo de un importante rey de Troya, que había sido supuestamente llevado por Zeus a los cielos cuando se personificó en la forma de un águila. Debido a esa interpretación de la autoridad del mito y la idea de la existencia de un deseo bastante fuerte y carnal por un hombre joven, ese cuento mitológico hace parte de algunos de las obras artísticas con connotación homo-erótica (ZANOTTI, 2007, p. 24).

En ciudades como Florencia y Venecia, la persecución a las personas homosexuales ha sido motivada por un gran incentivo económico a los habitantes que denunciaban a quienes realizaban tales conductas supuestamente condenables. Alder Izquierdo resalta que: 
Incluso los miembros de las órdenes religiosas sagradas debían informar a las autoridades de la más mínima sospecha, de no hacerlo, podían ser suspendidos de su sacerdocio. A su vez, a los confesos, se les prometía una pena menos y una recompensa si aceptaban convertirse en testigos de la acusación y denunciaban a sus compañeros (IZQUIERDO, 2013, p. 63).

Prácticas semejantes han sido seguidas por la Corona Portuguesa en las denominadas Ordenaciones del Reino Portugués, donde el incentivo a las denuncias de los casos movían a la sociedad de la época a la propia banalización de la incitación, es decir, denunciar en muchos casos solamente para denunciar y recibir ventajas económicas al hacerlo, sin que fuesen por ejemplos verdaderos.

Para Baile Ayensa, con el Renacimiento la visión de la homosexualidad cambió un poco en algunos países de Europa que pasaran por el movimiento histórico. Si bien que la acusación de sodomía siguió vigente, se relajó en cierto grado la persecución contra los hombres supuestamente homosexuales, y la pena no era inexcusablemente la muerte (AYENSA, 2008, p. 132). Es decir, el momento ha pasado por un ablandamiento de rigidez cuanto a las prácticas homosexuales, en su caso con penalizaciones menores que la pena capital.

En la Edad Media, las personas consideradas locas, eran los impíos, los lascivos y los sodomitas, como ejemplo de la época, citase el Arzobispo de Agobardo de Lyon en el Siglo IX, que ha luchado fervorosamente contra a los considerados indeseables como los idólatras y la brujería. De modo diverso, se presentó el Renacimiento, con una expresión de que la locura fascinará el mundo, recuperando de este modo las influencias de la cultura grecolatina, donde la supremacía de la belleza como un bien mayor y la búsqueda por placer caracterizaran esta manifestación histórica de la sociedad. Es válida para ese ejemplo, de los artistas homo-eróticos, una vez que para la elaboración de sus ideas, proyectos, artes, se basaran en la justificación de que sólo el amor real importaba y la naturaleza de su manifestación. La búsqueda por la belleza era lo más importante para tener en cuenta y la mayor expresión del alma (GARCÍA VALDÉS, 1981, p. 54).

También se puede atribuir a Leonardo da Vinci una supuesta acusación de práctica de sodomía, donde había tenido que comparecer al 9 de abril de 1476, con solamente 24 ańos de edad, junto a otros cuatro acusados ante los oficiales de la noche, con la sugerencia de haber realizado pecado nefando con un joven de 16 años (Jacobo Saltarelli) (GARCÍA VALDÉS, 1981, p. 54).

Para Alder Izquierdo:

En la medida que las creencias religiosas servían de base para un prejuicio particular, éste va a ser compartido por la mayoría de la población. Religión y prejuicios van de la misma mano y es en este momento histórico en donde los prejuicios acerca de las relaciones entre personas del mismo sexo se conectan con más fuerza, el nuevo occidente ve a las relaciones sexuales entre personas del mismo sexo con una extendida hostilidad pública, se empiezan a crear diversas restricciones civiles y legislaciones que alimentan cada vez más la homofobia, como también la ya existente discriminación social, todo con una justificación manifiestamente religiosa (IZQUIERDO, 2013, p. 62).

En relación al momento histórico, válidos en el ámbito más reciente la manifestación en el Siglo XX, más específicamente en la década de los 90, en Italia, la ARCI-GAY, que representaba la asociación italiana de los homosexuales, que después de una visita del fallecido Papa Juan 
Pablo II, al declarar que Miguel Ángel, otro destacado personaje histórico, había sido el mejor intérprete en sus obras sobre el cuerpo humano. Con relación a esos comentarios la asociación complementó en declaraciones a los medios de comunicación que: el formidable conocimiento del artista no se debia sólo a su genio de artista, sino también a su exquisita sensibilidad homosexual (PETIT, 2003, p. 239).

De acuerdo con Baile Ayensa:

Gran parte de la historia de la consideración de la homosexualidad es una historia de rechazo o de olvido. El rechazo se ha manifestado de múltiples maneras, desde acusarles de ser responsables de terremotos y pestes hasta perseguirles penalmente, pero también ha sido una triste historia de olvido; si bien a otros colectivos se les ha discriminado de igual o peor forma [judíos, mujeres...], a las personas homosexuales muchas veces se les ha negado la posibilidad siquiera de existir como tal realidad (AYENSA, 2008, p. 121).

Para Chaves, estas ideas serán usadas en casi todos los tipos de discursos contra la homosexualidad. Los distintos juicios de valor que se han formulado a partir del repertorio de unidades semánticas no neutrales, ya ciegas de ideología sistemática - que contienen la creencia en la existencia de una forma normal de práctica sexual: el coito entre un hombre y una mujer (CHAVES, 2009, p.36-7). Del mismo modo que argumenta:

El discurso social sobre la homosexualidad se basa en la construcción de una serie de nociones semánticas recortadas y moralmente sancionadas por la cultura sexual hegemónica. Expresiones aparentemente objetivas como "colectivo gay" o "comunidad homosexual" y la misma idea de homosexual han sido aceptas pasivamente por la sociedad y hacen parte de ideas que recrían en su interior una tabla de valores dominantes y lo respectivo sistema de relaciones sexuales socialmente legitimadas. Con la homosexualidad, la ética sexual clásica ensayó las hipótesis de la posesión satánica, el pecado y el delito y, hasta poco tiempo, la enfermedad. Hoy los homosexuales ya no son pecadores, ni delincuentes, tampoco enfermos, pero todavía sobrevive la definición: son distintos. En la historia de la homosexualidad hemos atravesado la era de la diferencia. Vamos ver que esta evolución considerada un progreso en el conocimiento y en la aceptación social de la homosexualidad, solamente es en aparente (CHAVES, 2009, p.31-2).

Con base en este entendimiento es válido recordar las argumentaciones de Chaves que enfatiza que esa creencia llevaría implícita la no aceptación de toda forma alternativa de relaciones sexuales entre personas del mismo sexo (CHAVES, 2009, p. 35). Para el autor, esta prohibición socialmente generalizada e individualizada es denominada tabú.

\section{La persecución a las personas homosexuales}

La cuestión de las persecuciones a los homosexuales se presenta como de relevo una vez que es actual y en distintas partes del mundo, violaciones a los derechos de esas personas siguen victimando a muchas personas debido a la manifestación de sus orientaciones sexuales como una actividad social (DA COSTA, 2012, 16). En algunos de estos casos citamos la situación en la zona de Asia y Pacífico, donde aportaciones informativas de la Organización de las Naciones Unidas estima que una media de 48 países de la región, entre ellos Bután, Kiribati, Malasia tienen previstos en sus Ordenamientos Jurídicos leyes que prohíben expresamente las conductas sexuales consentidas entre personas del mismo sexo (ANDRADE DO BOMFIM, 2011, p. 75- 
6).

No obstante, también encontraremos relatos de prácticas discriminatorias hacia a los homosexuales en el Continente Africado, con la criminalización expresa de la homosexualidad, que van desde a trabajos forzosos hasta la pena capital. Uno de los casos de relevo ha sido de la condena de un homosexual a 14 ańos con trabajos forzosos, donde la motivación de la sentencia condenatoria previa la terminología de práctica indecente grosera y actos no naturales (ANDRADE DO BOMFIM, 2011, p. 75-6).

En Uganda, discursos sobre la previsión del implementar la pena de prisión para los ciudadanos que no delataren a la policía a un homosexual, sin comentar la viabilidad de aplicación de la pena de muerte a los autores de práctica sexual entre homosexuales si alguno de ellos es portador del SIDA - síndrome de inmunodeficiencia adquirida. Otros casos de violaciones a los derechos de los homosexuales se notan en Burundi, país que también prevé, en sus normas, la condena al sexo homosexual consentido. Así como en Zimbabue, que el Gobierno, en 2010, obstaculizó cambios importantes en la Carta Magna del país, impendiendo poner límites a la discriminación hacia a ese colectivo. La excepción en el continente estaría en África del Sur, que adoptó la postura de retirar de su Ordenamiento Jurídico normas que fuesen discriminatorias a los homosexuales y implementó incluso el casamiento entre personas del mismo sexo (ANDRADE DO BOMFIM, 2011, p. 75-6).

Las ideas contrarias a las minorías se intensifican por el mundo, más específicamente en los países con una fuerte influencia de la religión (católica, ortodoxa, judaica, cristianismo, islamismo, evangélicos, etcétera). Es probable que debido a algunas ideas de estas religiones surgiera, de modo negativo, la comprensión de la homosexualidad en diferentes sociedades a lo largo de los tiempos (RIOS, 2009, p. 62).

Para Baile Ayensa:

La visión de la homosexualidad ha sido modificada progresivamente de manera que pasa el siglo XX, fundamentalmente en los países occidentales, sobretodo conforme avanzan los Estados con Cortes Constitucionales, donde se han protegido progresivamente las personas sin distinción de condiciones personales. Hecho eso posible después de las atrocidades de la Segunda Grande Guerra, donde el régimen nazista ha asustado la humanidad con sus barbaries contra los judíos, gitanos, minusvalorados y los homosexuales, entre otros tanto (Este progresivo respeto por la homosexualidad que se fue perpetuando en los países democráticos occidentales que han surgido con la Segunda Guerra Mundial, contrasta con la fuerte opresión a las que han sido obligadas las personas con la orientación del deseo homosexual en los sistemas autoritarios. Las dictaduras tienen un interese especial contra los homosexuales, sea a través de dictaduras de inspiración comunista (URSS, Cuba e otras), o por de inspiración capitalista e conservadora (a España de Franco, por ejemplo) (AYENSA, 2008, p. 135-6).

En realidad, la moralidad que los nazistas defendían era su instrumento de propaganda, poder y control de la población. También era un medio para facilitar y propagar una de las obsesiones del régimen: la política demográfica, uno de los mayores proyectos nazis. Las crianzas serían la generación natural de Alemania, donde un número alto de nacimientos significaría un flujo continuo de hombres para las fuerzas armadas. Esa nueva orden demográfica ha llevado el régimen a prohibir todo lo que dificultaba esa idea, criando incluso códigos morales especiales para las mujeres y para los homosexuales (BERTONHA, 2003, p. 49). 
Como dice Souto Galván:

La discriminación, como rasgo esencial de las ideologías nacionalistas, se hizo patente en la evolución del nacionalismo alemán que conjuró, sucesivamente, como enemigos de la nación alemana a los obreros, a los judíos, a los socialistas y a los polacos de la zona oriental de Alemania (GALVÁN, 2012, p. 40).

En relación a esas ideas añadiremos a la situación de minorías religiosas, como eran los testigos de Jehová y también a los homosexuales, que han sido victimados por la barbare del Régimen.

Para complementar esa idea aducimos que el Sistema Nacional-Socialista no criticaba la homosexualidad por su aspecto moral, pero por sus razones extremadamente prácticas, como bien nos aclaraba Bretonha. Según el autor: el objetivo del sexo es la reproducción y el hombre utiliza esa energía sexual en aventuras con otro hombre, obviamente, solo se podrá generar hijos de estas prácticas. De este modo sería considerado como a un criminoso contra el Reich y debería ser punido por estos hechos (BERTONHA, 2003, p. 49).

Durante la vigencia del sistema Nacional-Socialista en Alemania, período de la Segunda Grande Guerra, los homosexuales masculinos y femeninos perseguidos por el sistema y que habían sido encaminados para los campos de concentración, usaban en sus vestimentas un triángulo rosa, justamente para identificarlos como homosexuales y sofrieren humillaciones por sus orientaciones del deseo. Estos actos eran comunes en aquel período histórico. Después de esta Guerra, el triángulo rosa se tornó símbolo del movimiento del orgullo homosexual (VERBICARO SOARES, 2004, p. 14-5). Para Raupp Rios: la homofobia nazista, preocupada con la expansión de la población arriana y la supremacía alemana, se valido de bases biológicas y morales para condenar y conducir por lo menos 500.000 homosexuales a la muerte en las prisiones (RIOS, 2009, p. 63). Petit también ha mencionado ese período histórico:

La persecución de los homosexuales por el nazismo se justificaba en base a la pureza de la raza arriana, y poco se sabe de lesbianas detenidas. Hasta 50.000 homosexuales llegaron a ser arrestados en Berlín y se calcula que cerca de medio millón en los campos de exterminio, lo que hace jus a los argumentos presentados anteriormente. Las personas homosexuales eran marcadas con un triángulo rosa, símbolo y color que luego adoptó el moderno movimiento de gais y lesbianas (PETIT, 2003, p. 140).

En lo que se relaciona con las lesbianas, prostitutas, mujeres sin niños y aquellas con algún tipo de peculiaridad anti-social, que no se encuadran en el padrón esposa-madre-ama de casa. Fue durante la Segunda Grande Guerra que el Sistema Nazista de Hitler, ha sellado con un triángulo negro estas mujeres en los campos de concentración (ABGLT, 2010, p. 41).

La historia muestra que la humanidad sigue marcada por variados momentos de abusos a los derechos de las minorías. Tal vez una de las teorías que se han buscado para justificar las violaciones contra los homosexuales estaría en el riesgo de que un no aumento de la procreación humana de una sociedad. El Sistema nacional-socialista alemán sería un grande problema para la consolidación del mismo sistema totalitario. De esta forma, las prácticas homosexuales eran consideradas como una pérdida de tiempo, pues biológicamente imposibilitaban la generación de crianzas y de la continuidad de la raza alemana (VERBICARO SOARES, 2004, p. 14-5).

Con base en ese raciocinio utilizaremos las ideas de Raupp Rios: 
En este contexto, una hipótesis particularmente divulgada es la de que las relaciones homofóbicas violentas provienen de sujetos en grave conflicto interno con sus propias tendencias homosexuales, resultantes de la proyección de un sentimiento no soportable de identificación inconsciente con la homosexualidad. No obstante, la discusión freudiana delante de la homosexualidad, el hecho de que la "homofobia clínica", al lado de la "homofobia antropológica", del estalinismo y del nazismo han sido las principales ideologías que construyeran la homofobia moderna, de carácter laico y no-teológico (RIOS, 2009, p. 62).

En este sentido nos viene a la mente la justificativa que hemos encontrado relacionada a la idea de que como los homosexuales no pueden perpetuar la especie, es decir, dar continuidad biológica a la sociedad, esa justificación incide de modo negativo sobre la visión de la homosexualidad. La persecución de los regímenes totalitarios hacia a esa orientación sexual, han generado incluso reglamentos específicos que cohibían, con la muerte, las personas que no se adecuasen a los padrones previamente establecidos, en este caso el heterosexual dominante en sociedad (VERBICARO SOARES; CRUZ, 2018, p. 304).

A los homosexuales se les atribuía a categorías de enfermos (TROIDEN, 1988, p. 125), anormales, pecadores y antinaturales (ELÍAS, 2007, p. 202), los más terribles adjetivos que podían ser conferidos a una persona (AYENSA, 2008, p. 126-7). De todos los modos, después de la formación de ese ambiente hostil en el sentido negativo que caracterizaba a las atrocidades cometidas por el Régimen totalitario y las conductas completamente contraria al reconocimiento de la dignidad humana, surge la necesidad internacional en plasmar de un modo universal ciertos derechos a ser protegidos a toda labor. Los Derechos Humanos se plasman en esa esfera histórica.

\section{Consideraciones finales}

Frente a la visibilidad histórica sobre la homosexualidad en el tiempo, distintos momentos han retratado la condena de la diversidad sexual y en el no reconocimiento de sus manifestaciones en diversas sociedades por el mundo. Desde la condena por medio de Ordenamientos legales como explicitados en esa investigación, como en las Ordenaciones Portuguesas, que han influido socialmente en la moralidad de la religión Católica por el Reino de Portugal y sus colonias en la Edad Media, o a través de la persecución de los homosexuales por el Régimen del Nacional Socialista en el siglo XX, incluso en la presencia de actuales Ordenamientos Jurídicos que refuerzan la condena por prácticas afectivas/sexuales entre personas del mismo sexo por ciertos países.

Así, la historia repite roles discriminatorios que condenan la sexualidad humana. Todo lo que es diverso de la heterosexualidad normativa dominante y reproductiva auxilia en la exclusión de las personas que no siguen eses mismos roles restrictivos. El desafío de la educación para los Derechos Humanos y en la efectiva igualdad y aceptación de las personas homosexuales necesita otros esfuerzos para cambiar los obstáculos que impiden la real integración de los excluidos.

\section{Referencias}

ABGLT. Manual de Comunicação LGBT. Lésbicas, Gays, Bissexuais, Travestis e

Transexuais. Brasília, Brasil, 2010. 
ANDRADE DO BOMFIM, Silvano. Homossexualidade, Direito e Religiáo: pena de morte à uniáo estável. A criminalizaçáo da homofobia e seus reflexos na liberdade religiosa. In: Revista Brasileira de Direito Constitucional - RBDC n. 18 - jul./dez. Brasil, 2011.

AYENSA, José Ignacio. Estudiando La Homosexualidad. Teoría e investigación. $1^{a}$ Edición, Ediciones Pirámide. Madrid, España, Ediciones Pirámide, 2008.

BERTONHA, João Fábio. Fascismo, nazismo, integralismo. História em movimento. $1^{\text {a }}$ edição. Editora Ática. São Paulo/SP, Brasil, 2003.

DA COSTA, Rogério. Homossexualidade: um conceito preso ao tempo. In.: Bagoas - Estudos gays: gêneros e sexualidades, v. 1, n. 01, 27 nov. 2012. Disponible: <https:// periodicos.ufrn.br/bagoas/article/view/2255>. Acceso en: 10 mar. 2021.

ELÍAS, Cristina. Cultura Homosexual, Sujeto. Homosexual y Derechos Humanos. In: FORO. Revista de Ciencias Jurídicas y Sociales, Ensayos, No 5. Nueva Época. Madrid. España, 2007.

GALVÁN, Clara. Principio de igualdad y transversalidad de género. Editora Dykinson. Madrid, España, 2012.

GARCÍA VALDÉS. Alberto. Historia y Presente de la Homosexualidad. Editorial Akal/ Universitaria, Madrid, España, 1981.

IZQUIERDO, Alejandro. Realidad jurídica y social del derecho a la orientación e identidad de género. Tesis Doctoral. Universidad de Salamanca: Programa de Doctorado Pasado y presente de los derechos humanos, Salamanca, España, 2013.

CHAVES, Norberto. La homosexualidad imaginada. Vigencias y ocaso de un tabú. Maia Ediciones. Madrid, España, 2009.

PETIT, Jordi. 25 años más. Una perspectiva sobre el pasado, el presente y futuro del movimiento de gais, lesbianas, bisexuales y transexuales. $1^{a}$ Edición. Icaria Editorial. Barcelona. Cataluña, España, 2003.

RIOS, Roger. Homofobia na perspectiva dos direitos humanos e no contexto dos estudos sobre preconceito e discriminaçáo. In: Diniz Junqueira, Rogério: Diversidade Sexual na Educação: problematizações sobre a homofobia nas escolas. Coleção Educação para todos. Brasília, Brasil, 2009.

TROIDEN, Richard. Gay and Lesbian Identity. A sociological analysis. Miami University. Oxford, Ohio. EE. UU. 1988.

VERBICARO SOARES, Douglas; CRUZ, Rivetla. Históricas influências artísticas na formaçáo de ideários sobre a orientaçáo homossexual na sociedade brasileira. In.: Revista Pensamento Jurídico - FADISP. Vol.12, no 2, jul./dez., p. 281-307, 2018.

VERBICARO SOARES, Douglas. O Sistema Nacional-Socialista e suas formas de repressáo às minorias. Totalitarismos e a efetividade dos Direitos Humanos. Trabalho Final de Graduação em Direito. Universidade Federal do Pará/UFPA. Belém, Brasil, 2004.

ZANOTTI, Paolo. Gay. La identidad homosexual de Platón a Marlene Dietrich. Colección Noema. Turner Publicaciones. Madrid, España, 2007. 